\title{
Griechenland nach den Wahlen
}

Nach den Wahlen vom 25. Januar 2015 und der Bildung der neuen Koalitionsregierung flammt die Debatte über die Zukunft Griechenlands erneut auf. Die Partner der neuen griechischen Regierung gewannen die Wahlen aufgrund ihrer Versprechungen, die Austeritätspolitik zu beenden, das von der vorherigen Regierung mit der Troika vereinbarte Reformprogramm zu stoppen und eine neue Vereinbarung mit den europäischen und internationalen Gläubigern über einen Schuldenerlass auszuhandeln. Ferner haben sie versprochen, viele von den bereits realisierten Reformen in den Bereichen Arbeitsmarkt, Versicherung, Bildung, Verwaltung, Banken, Privatisierung und andere aufzuheben. Durch zusätzliche Ausgaben sollten neue soziale Maßnahmen finanziert werden. Nach ihrem Amtsantritt hat die neue Regierung vier politische Prioritäten gesetzt: (1) das Brechen des rezessiven Teufelskreises und die Förderung des Wirtschaftswachstums, (2) die Bewältigung der akuten sozialen Probleme, (3) die Schuldenerleichterung und die Anwendung eines eigenständigen nationalen Reformprogramms mit Schwerpunkten auf der Verbesserung der Staatsverwaltung, (4) der Beseitigung der Steuerhinterziehung, der Bekämpfung der Korruption und der Beendigung der Klientelpolitik. Es wurde allerdings betont, dass Griechenland in der Eurozone bleiben werde. Die Regierung beabsichtigt jedoch nicht, den eingegangenen Verpflichtungen nachzukommen. Die Fragen der Schulden, der Zinszahlungen, der Bankenliquidität, des Wachstums und der Reformen will sie im Rahmen einer neuen europäischen Vereinbarung und ohne die bisherige Überwachung der Troika regeln. Bis zur Erreichung einer endgültigen Vereinbarung mit den Europartnern und dem Internationalen Währungsfonds (IWF) wird ein „Überbrückungsprogramm“ vorgeschlagen. Zur Deckung der bevorstehenden Kredittilgungen an den IWF und die Europäische Zentralbank (EZB) und der Zinszahlungen will die Regierung keine weiteren Kredittranchen beanspruchen, sondern diese durch kurzfristige Kredite und die Überweisung der Gewinne aus griechischen Staatstiteln der nationalen Zentralbanken des Eurosystems finanzieren. Wegen der heftigen Reaktionen der Gläubigerstaaten hat die griechische Regierung ihre These über einen weitgehenden Schuldenschnitt zurückgenommen; nun spricht sie über eine Umschuldung mittels neuer Finanzinstrumente. Die Regierung sieht die vorgesehenen primären Haushaltsüberschüsse zur Deckung der Zinszahlungen als zu hoch an und als ein Hindernis dafür, die Wirtschaft anzukurbeln. Sie zielt auf eine Senkung der Überschussquote von $4 \%$ bis $5 \%$ auf $1 \%$ bis $1,5 \%$ des Bruttoinlandsprodukts (BIP), damit die Binnennachfrage und die öffentlichen Investitionen gestärkt werden.

Ausschlaggebend ist jedoch nicht nur, was die neue griechische Regierung anstrebt, sondern was die Gläubiger (Euroländer, EZB, IWF) zu akzeptieren bereit sind. Aus den bisherigen Reaktionen und Stellungnahmen wird ersichtlich, dass die meisten griechischen Vorschläge auf Ablehnung stoßen. Nach Meinung der Gläubiger solle Griechenland sich an die eingegangenen Verpflichtungen, wie sie im zweiten Memorandum verankert sind, halten. Weitere Kredittranchen würden nur nach Vorlage eines positiven Evaluierungsreports der Troika gewährt. Griechenland solle die vereinbarten Reformen durchführen und die geplanten primären Haushaltsüberschüsse erreichen. Bisherige Reformen seien nicht anzutasten und Wahlversprechen, die vom geltenden Anpassungsprogramm abweichen, dürften nicht realisiert werden. Zusätzlich solle die neue Regierung den Zahlungsverpflichtungen gegenüber den Gläubigern nachkommen und die institutionellen Spielregeln der Eurozone respektieren. Das Gleiche gelte für die Liquiditätsversorgung der griechischen Banken von der EZB. Letztere hat bereits angekündigt, dass griechische Staatspapiere nicht mehr als Sicherheit für neue EZB-Kredite genutzt werden. Kurzfristig können sich griechische Banken teureres Geld durch den ELA-Mechanismus (Emergency Liquidity Assistance) verschaffen. Ein neues Hilfsprogramm wäre nur möglich, wenn Griechenland im Rahmen des neuen Programms Gegenleistungen erbringe, die weiterhin von der Troika überwacht würden.

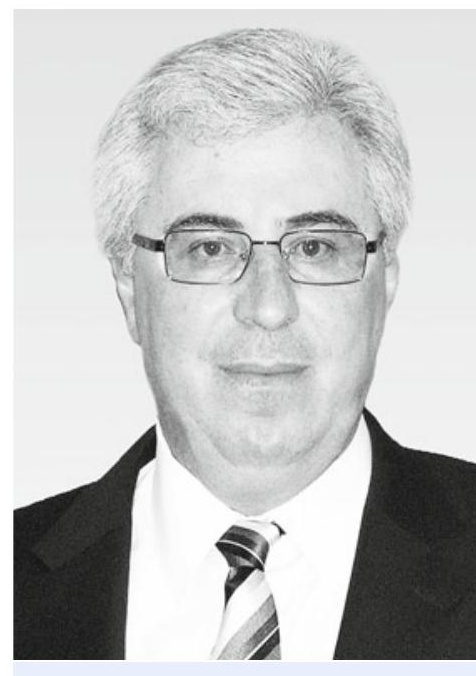

Angelos Kotios ist Lehrstuhlinhaber für Internationale und Europäische Wirtschaftspolitik an der Universität von Piräus, Griechenland. 
Die Positionen weichen also weit voneinander ab. Würde jede Seite auf ihrer Position beharren, wäre eine Konfrontation unvermeidbar. Dies hätte katastrophale Folgen für Griechenland, aber auch negative Auswirkungen auf die Eurozone und auf die internationale Wirtschaft. Griechenland hätte keinen Zugang zur Außenfinanzierung, um seine Staatsanleihen zu bedienen. Das Land wäre dann offiziell bankrott. In diesem Fall müsste es mit Folgen wie Sanktionen, politischer Isolation, langjähriger Abkopplung von internationalen Kapitalmärkten sowie mit gerichtlichen Verfahren rechnen. Im Innern des Landes wären ein Bank Run, massive Kapitalexporte und - wegen der Refinanzierungssperre der Banken von der EZB - eine totale Liquiditätsdrosselung zu erwarten. Eine solche Entwicklung würde die reale Wirtschaft und den sozialen Zusammenhalt zerstören. Demzufolge wäre mit politischen Konfrontationen, weiterer Polarisierung der Gesellschaft, sozialen Unruhen und der Demontierung des Staatsapparats und des organisierten Lebens zu rechnen. In einer solchen Situation wären der Austritt aus der Eurozone und der Übergang zur nationalen Währung, um die Liquiditätsengpässe zu überwinden, sehr wahrscheinlich. Ein Bankrott und ein möglicher Euroaustritt Griechenlands hätten aber Ansteckungseffekte auf die anderen Euroländer, insbesondere auf die wackelnden südeuropäischen Länder, obwohl die Risiken aufgrund der Etablierung des Europäischen Stabilitätsmechanismus (ESM) und der beschlossenen expansiven Geldpolitik (Quantitative Easing) der EZB mittlerweile für beherrschbar gehalten werden. Ferner könnte diese Entwicklung das Vertrauen in den Zusammenhalt der Eurozone und die internationale Attraktivität des Euro schwächen. Zusammenfassend lässt sich feststellen, dass ein Konfrontationskurs für alle Seiten schädlich wäre. Es handelt sich um ein „lose-lose game“, unabhängig davon, dass Griechenland offensichtlich viel mehr als die übrigen Euroländer zu verlieren hat. Durch Kompromissbereitschaft sollten neue Verhandlungen zu einem „Win-win-Ergebnis“ führen. Dazu sind schwerwiegende Entscheidungen, die politische Auswirkungen nach sich ziehen würden, zu treffen.

Die neue griechische Regierung sollte ihre populistischen Taktiken und viele Wahlversprechungen, die sowieso nicht finanzierbar sind, aufgeben. Sie muss auch anerkennen, dass internationale Vertragsbindungen nicht durch Wahlen einseitig annulliert werden können. Alte Verträge dürfen nur durch neue novelliert werden. In diesem Sinne sollte sie ein realistisches neues Reform- und Wachstumsprogramm ausarbeiten und es dann den Gläubigern vorlegen. Durch dieses Programm könnten endlich die tieferen Ursachen der Krise wie Korruption, Steuerhinterziehung, Klientelwirtschaft, Bürokratie, mangelnde Wettbewerbsfähigkeit usw. bekämpft werden. Das Land soll die Eigenverantwortung für seine Strukturreformen übernehmen. Durch institutionelle Reformen und ordnungspolitische Maßnahmen muss Griechenland eine gesunde Wettbewerbswirtschaft mit offenen Märkten etablieren und die Rahmenbedingungen für ausländische Investoren verbessern. Wie die Erfahrungen anderer Krisenländer zeigen, sind massive Direktinvestitionen und Privatisierungen notwendig, um hohe Wachstumsraten zu erreichen. Beim Problem der Schulden besteht wenig Raum zu Kursänderungen. Gesunde Staatsfinanzen und primäre Haushaltsüberschüsse sind grundsätzlich notwendig, um eine neue Verschuldung zu vermeiden. Die Kredittilgungen bis 2020 sind relativ gering und betreffen nur die Kredite des IWF und der EZB. Für eine Verlängerung der Kreditlaufzeiten und eine weitere Senkung der Zinsen besteht beschränkter Handlungsspielraum. Um das Wachstum des Landes zu fördern, was die späteren Zahlungsverpflichtungen sichern würde, könnten Zinszahlungen an das Wirtschaftswachstum gekoppelt werden. Um dem Land eine Atempause zu gewähren, wäre auch zu prüfen, ob die ausstehenden Schuldanleihen des IWF und der EZB durch Anleihen des ESM ersetzt werden könnten. Die europäischen Partner sollen ihrerseits anerkennen, dass das angewandte wirtschaftspolitische Anpassungsprogramm und die intensive Austeritätspolitik sehr hohe politische, soziale und ökonomische Kosten verursacht haben. Es fehlten ein direktes Wachstumsziel bzw.

Angelos Kotios

Universität Piräus

akotios@gmail.com wachstumsfördernde Maßnahmen, sowie flankierende sozialpolitische Stützungsmaßnahmen. Ohne eine Unterstützung der Europartner kann Griechenland aus dem Teufelskreis der wirtschaftlichen und sozialen Depression nur sehr schwer herauskommen. 